\title{
Development and application of intermittent electron irradiation technique with a high voltage electron microscope
}

\author{
S. $\operatorname{Arai}\left({ }^{1}\right)$, N. Yokoi $\left({ }^{1}\right)$, C. $\operatorname{Morita}\left({ }^{1}\right)$ and M. Kiritani $\left({ }^{2}\right)$ \\ ( ${ }^{1}$ 1MV Electron Microscopy Laboratory, Nagoya University, Furo-cho, Chikusa-ku, Nagoya 464, \\ Japan \\ (2) Department of Nuclear Engineering, Nagoya University, Furo-cho, Chikusa-ku, Nagoya 464, \\ Japan
}

(Received 15 March 1993, accepted 27 April 1993)

\begin{abstract}
For the purpose to observe and analyze the transient phenomena after the start of irradiation and the relaxation phenomena after the cessation of irradiation, techniques for intermittent electron irradiation are developed with a high voltage electron microscope, utilizing the STEM function installed in H-1250ST. One of the two methods developed is to make move the electron beam along a circle passing the area of interest in each cycle, which has an advantageous feature of invariant accumulated irradiation time regardless of the frequency. In another method, the electron beam goes back and forth between two positions, in which there is a free choice of the combination of the on-and off-time of electron beam. Dynamical control of electron beam position is made with two deflection coils placed at a right angle, which are driven by a computer loaded with two-phase-signal oscillator through D/A converter. Examples of applications are quoted on the relaxation effect of accumulated point defects on the nucleation and growth of interstitial clusters introduced in metals by electron irradiation.
\end{abstract}

\section{Introduction.}

High voltage electron microscopes (HVEM) have been used widely in the experimental studies of crystalline defects, efficiently utiizing regarding the displacement of atoms by the collision of energetic electrons [1]. Extracting the fundamental properties of interstitial atoms and lattice vacancies, which are the basic components of variety of solid state reactions, the study using HVEM has been contributing to the progress of a wide field of material science [2]. Moreover, the understanding of the mechanism of damage structural developments under the introduction of simple defects has been contributing to the studies of more complicated defect processes such as ion implantation and neutron irradiation, giving a basis for the development of new materials [3].

These continuing of HVEM comes from its superior functions. The first is in its high efficiency of defect introduction [4]. For example, the production rate of Frenkel pairs is 10,000 times more than in the fuel-core position of a powerful nuclear reactor. The second is in its ability of direct 
observation and recording of dynamical defect reaction processes. This in-situ observation has been serving as the basis for the progress of qualitative and quantitative studies. The third is in its easy and accurate control of various experimental conditions, for example, electron irradiation intensity, specimen temperature and crystal orientation, etc.

In order to enhance the superior ability of HVEM, a technique of electron beam control for intermittent irradiation has been developed. It has a possibility to extract the phenomena which are difficult or impossible to observe under the continuous irradiation.

\section{Design of the control of electron beam for intermittent irradiation.}

A high voltage electron microscope $\mathrm{H}-1250 \mathrm{ST}$ at Nagoya University has functions of both scanning transmission electron microscopy (STEM) and conventional transmission microscopy (CTEM) [5]. Using this advantageous features, it is possible to perform the observation of structural changes by electron irradiation with its CTEM, and to control electron beam with scanning coil of STEM. By applying controlled signal to the scanning coil, electron irradiation can be repeated intermittently; the cyclic repetition of the irradiation and un-irradiation.

In order to perform a systematically repeated irradiation, two different types of electron beam control, the circle beam method and flip-flop beam method, were designed and the hard and soft wears required will be explained.

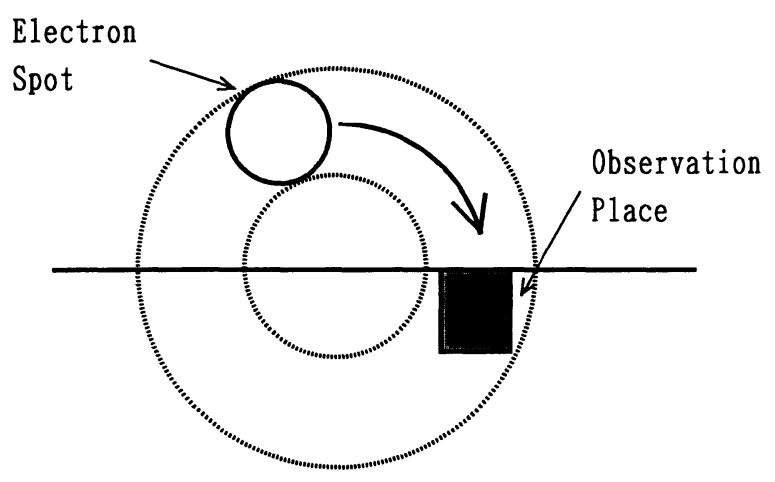

Fig. 1. - Schematic illustration of circle beam method. Electron beam makes circle and intermittently paths the observation area.

\subsection{CHARACTERISTIC FEATURES OF TWO METHODS.}

2.1.1 Circle beam method - Figure 1 shows the motion of electron beam with reference to the observation area in the circle beam method. The electron beam spot moves along a circle with designed radius and cycle. The beam is rotated by the combination of the deflection with $X$ and $Y$ scanning coils, being driven by sinusoidal signals with 90 degrees difference in phase. The advantage of this method lies in the fixed ratio of on-time and off-time of electron beam regardless of frequency. In another word, the accumulated irradiation time is proportional to the total of experimental time. The ratio can be changed by changing the size of circle and/or the size of electron beam spot. 


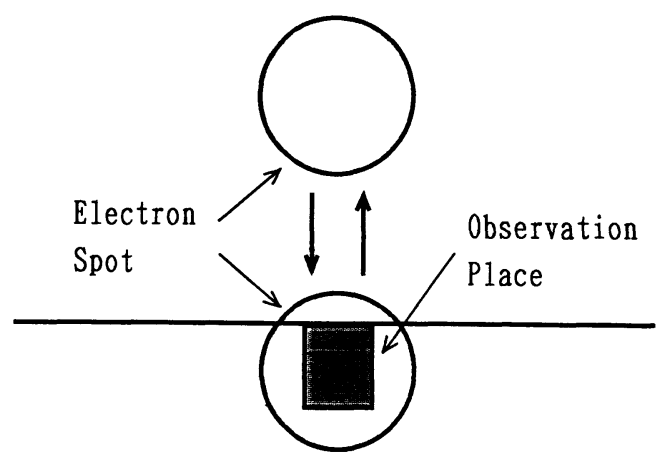

Fig. 2. - Schematic illustration of flip-flop beam method. Electron beam comes back to the observation area intermittently.

2.1.2 Flip-flop beam method - Figure 2 illustrates the motion of electron beam in flip-flop beam method. In this method, the electron beam spot moves between two positions back and forth. The deflection of beam is done by the pulse signal on $X$ and $Y$ deflection coils. The direction and the amount of the deflection can be changed by changing the strength and ratio of pulse signals on $X$ and $Y$ deflection coils. The advantage of this method is in the independent free choice of on-time and off-time.

2.2 REQUIRED PERFORMANCE OF THE ELECTRON BEAM CONTROL - As typical examples of the phenomena during the transition process after the cessation of irradiation and the relaxation process after the start of irradiation, the point defect cluster formation introduced by electron irradiation is adopted. From the size and the number density of defect clusters, the suitable magnification and electron spot size are $1 \sim 10 \times 10^{4}$ and $1 \sim 10 \mu \mathrm{m}$, respectively. For the required distance of the electron beam spot movement at the specimen position to be several times of the spot size, the current to control the scanning coil was determined $(0.1 \sim 5 \mathrm{~mA}$ for H-1250ST). Another requirement is the wide range of cycles; the wide range of the combination of irradiation time (on-time) and un-irradiation time (off-time). Temporarily, $0.01 \mathrm{~Hz} \sim 1 \mathrm{kHz}$ as the cycle, i.e. $100 \mathrm{~s} \sim 1 \mathrm{~ms}$ per one cycle were aimed at. Requirements are summarized as follows.

1) In addition to the wide range of cycles $\left(\geq 10^{5}\right)$, the ratio of on-off times must be varied widely, e.g. the combination of $1 \mathrm{~ms}$ on-time and $100 \mathrm{~s}$ off-time, the ratio of $10^{5}$.

2) In order to have a short switching time (such as $1 \mathrm{~ms}$ ), time duration of deflection of electron beam should be much less than the switching time $(\geq 10 \mu \mathrm{s})$.

3) In any combination of on-time and off-time, the electron beam spot must come back exactly at the same position.

The description above is based on one of the two methods developed in this paper which adopts the simple intermittent change of electron beam position. The control of the motion of electron beam in the circle beam method is easier, and a higher frequencies such as $100 \mathrm{kHz}$ can be achieved.

\section{Driving system of beam deflection coils and performance of beam control.}

The control of electron beam above mentioned could not be done by using a usual frequency oscillator, because of so wide required ratio of on- and off-time in addition to another wide range 
of required frequencies. A computer program was designed for frequency control and a driving unit for scanning coils was constructed.

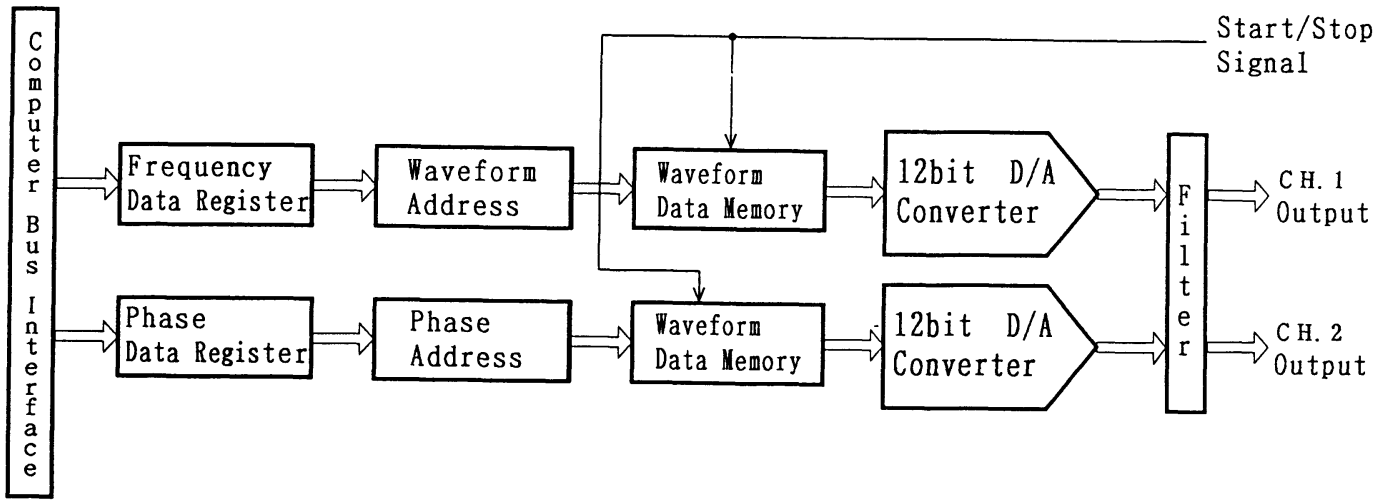

Fig. 3. - Functional units in the two-phase signal oscillator board.

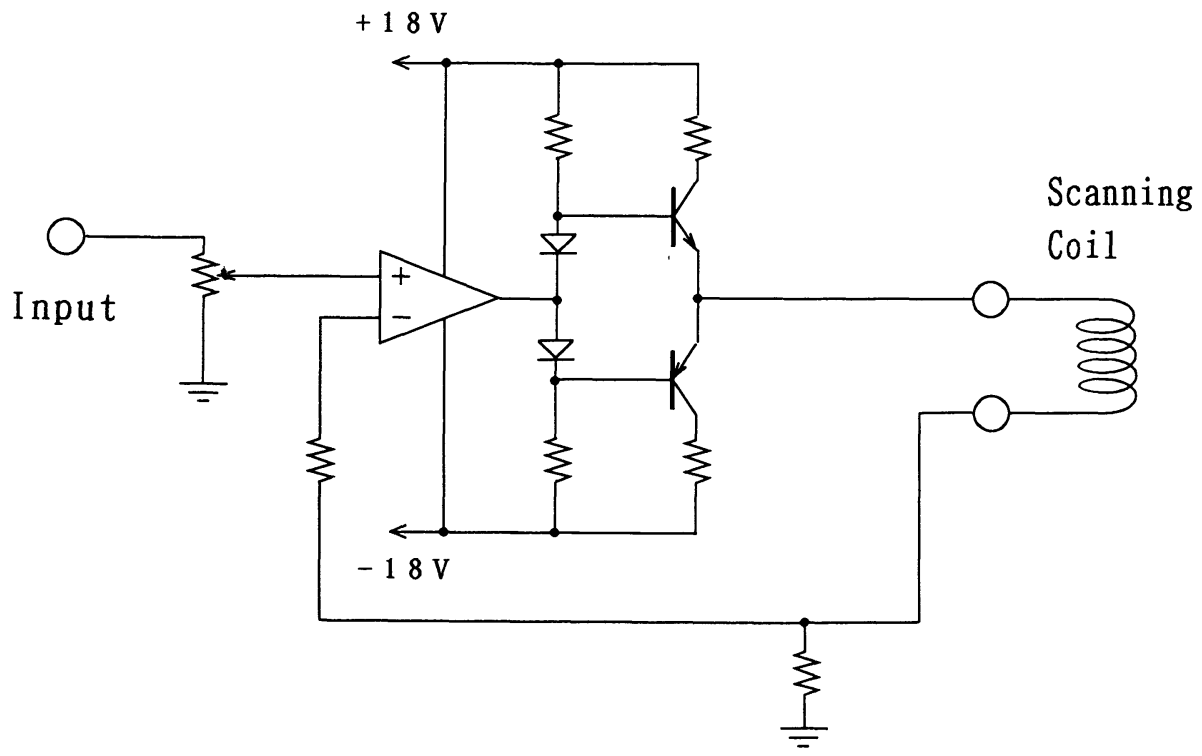

Fig. 4. - Coil current-feedback circuit for the compensation of the change of reactance with frequencies.

3.1 TwO-SIGNAL OSCILLATOR BOARD - Both for the circle and flip-flop beam methods, it is necessary to generate two signals having a designed phase difference. Figure 3 shows the block diagram of two-phase signal oscillator board. Signal generated are stored in waveform data memories, and the wave data are converted to analog output through the 12 bit D/A converter. The 
reason why 12 bit is required is to obtain a smooth wave shape, e.g. $2.4 \mathrm{mV}$ per 1 bit in the case of $\pm 5 \mathrm{~V}$ full scale. The wave data memory contains the function to stop the signal at the definite phase position after receiving the stop sign, in order to make stop the beam spot at exactly the same position as it started.

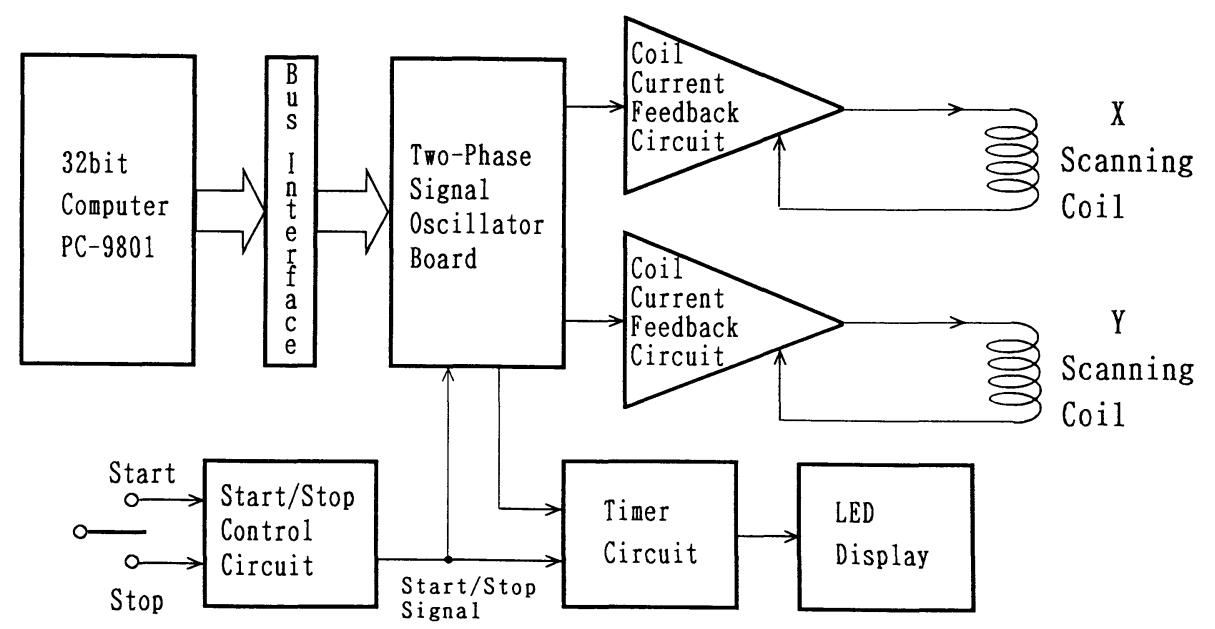

Fig. 5. - Total diagram of the driving system for two scanning coils.

3.2 COIL CURRENT-FEEDBACK AND CONTROL OF BEAM DEFLECTION - When the deflection coils are operated at a fixed frequency such as in the normal STEM mode, the variation of the reactance with frequency does not bring any serious problem. In the present case however, the change of the amount of beam deflection with frequency because of the increase of the coil reactance (decrease of deflection into one-half of $100 \mathrm{~Hz}$ at $1 \mathrm{kHz}$ ) should be avoided. In order to have the same deflection of the beam regardless of frequency, a current-feedback circuit using high-speed OP amp. for deflection coil as shown in figure 4 was inserted between the two-phase signal oscillator board and coils as shown in figure 5. The timer circuit in the total diagram in figure 5 is operated by the start/stop control circuit and LED display is accompanied, and the accumulated irradiation and/or the total experimental time can be measured and displayed.

3.3 PERformance of THE EleCtron BEAM CONTROL - The variation of electron beam flux on the observation area was measured with a Faraday cup placed at the position between the projection lens and the fluorescent screen. Figures 6 and 7 are examples of the circle beam method and the flip-flop beam method, respectively. The former is an example of shorter beam on-time than off-time, and the later is an example of opposite combination. Frequencies in these examples are almost at the highest frequencies described in 2.2 .

\section{Examples of application.}

Two examples of intermittent electron irradiation technique, a circle beam method and flip-flop beam method, are quoted. Detailed analyses of radiation induced point defect reactions are not 


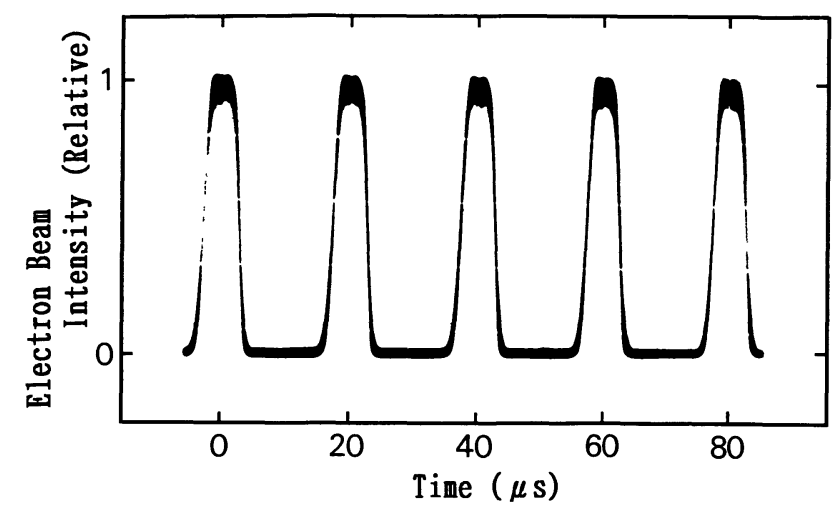

Fig. 6. - An example of electron beam intensity variation measured with Faraday cup in circle beam method.

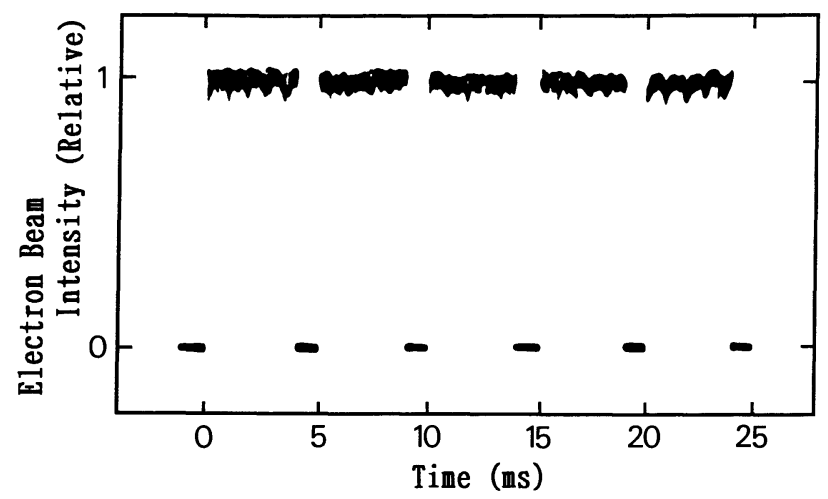

Fig. 7. - An example of electron beam intensity variation measured with Faraday cup in flip-flop beam method.

described, because the purpose of this paper is to report the development of the technique of intermittent electron irradiation.

4.1 AN EXAMPLE OF CIRCLE BEAM METHOD - Interstitial atoms produced by electron irradiation aggregate and form interstitial type dislocation loops. It is known that their growth is controlled by the motion of vacancies and their variation with experimental parameters has been used for determining the vacancy mobility [6]. These researches have been based on the analysis of experimentally measured loop growth speed under steady state irradiation. The balance relation between vacancies and interstitial should be further examined by the sudden change of irradiation condition. The intermittent irradiation technique developed in this paper has been applied to emphasize the effect after the cessation of irradiation by repetition.

Figure 8 shows the variation of the growth speed of interstitial loops as a function of off-time with a fixed on-time (the ratio of on-time to off-time is about 0.2 in this case of aluminum). The growth speed was observed to vary strongly at $100 \mathrm{cycles} / \mathrm{sec}$ (the combination of $2 \mathrm{~ms}$ on-time and $8 \mathrm{~ms}$ off-time). Skipping the detail of analysis, the bordering off-time indicates the time of escape of vacancies to sample surfaces, and can be utilized for the measurement of vacancy mobility. 


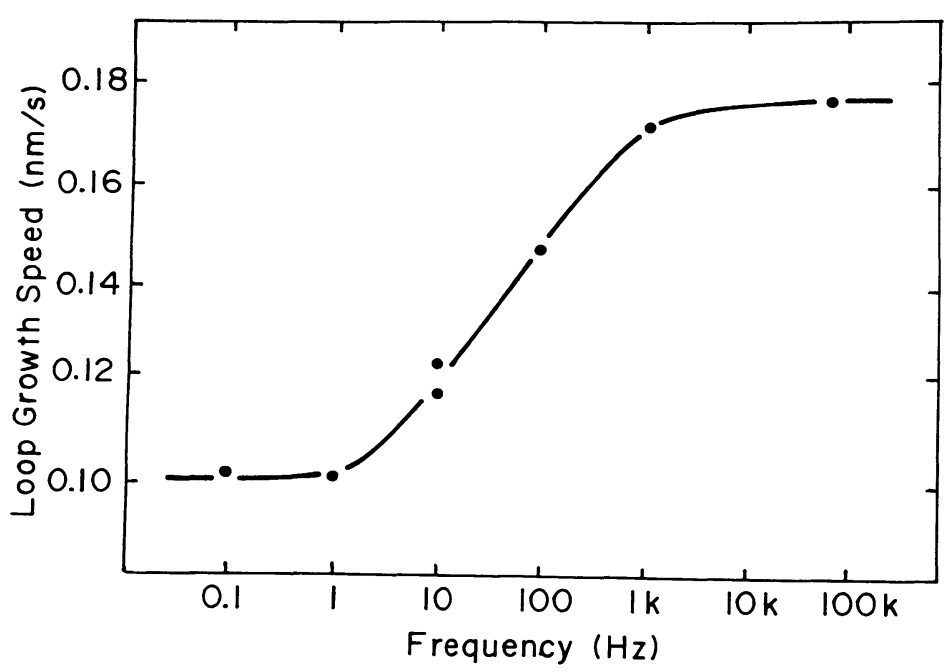

Fig. 8. - Variation of loop growth speed with the frequency in intermittent electron irradiation of aluminum. Irradiation with circle beam method. On- and off-time ratio of the electron beam was about 0.2 .

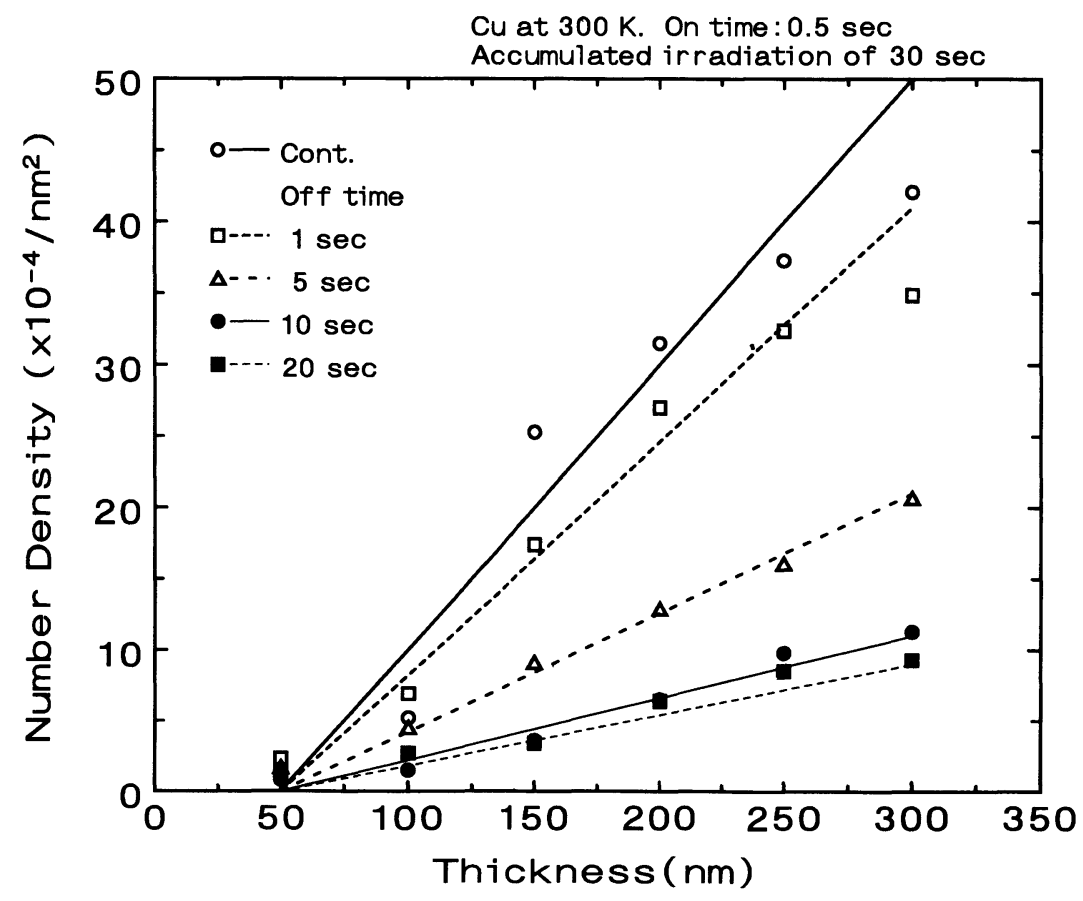

Fig. 9. - Variation of the nucleation of interstitial type dislocation loops with the electron beam off-time with fixed on-time $(0.5 \mathrm{~s})$. Number of loops per volume which is not affected by specimen surface can be measured from the gradient of the areal number density along the increase of specimen thickness. 
4.2 AN EXAMPLE OF FLIP-FLOP BEAM METHOD - The nucleation of interstitial clusters by electron irradiation has been known to finish by a short duration after the start of irradiation $(\leq 0.1$ s) [7]. An example of experiments to know the point defect reaction during this short duration is shown in figure 9. The figure shows the variation of the number density of interstitial clusters with the change of off-time with a fixed on-time. The volume number density which is not affected by the surfaces can be obtained as the rate of increase of areal number density with the increase of thickness of a wedge shaped specimen. The number of interstitial clusters is found to decrease drastically with an increase of electron beam off-time. It cannot be definitely stated at present that the decrease of the nucleation of interstitial clusters is due to the relaxation of reaction of vacancies accumulated during irradiation or due to the repetition of the transient phenomena occurring during the short duration after the start of irradiation.

\section{Acknowledgements.}

The authors are grateful to Professor M. Hibino, the head of Electron Optics Laboratory, and Professor H. Saka, the chairman of the executive committees of 1 MV HVEM Laboratory, for their continuous encouragement.

\section{References}

[1] KIRITANi M., Electron Microsc. (Bulltain of Japanese Society of Electron Microscopy) 27 (1992) 101.

[2] KIRITANI M., Ultramicroscopy 39 (1991) 135.

[3] KIRITANI M., J. Nucl. Mater. (1993) in press.

[4] KIRITANI M., J. Nucl. Mater. 169 (1989) 89.

[5] Hibino M., SHImOYAMA H. and MARUSE S., J. Electron Microsc. Tech. 12 (1989) 269.

[6] Kiritani M., Yoshida N., TaKata H. and Maehara Y., J. Phys. Soc. Jpn. 38 (1975) 1677.

[7] Yoshida N. and KiRITANi M., J. Phys. Soc. Jpn. 35 (1973) 1418. 